All homogeneous pure radiation spacetimes satisfy the Einstein-Maxwell equations

This article has been downloaded from IOPscience. Please scroll down to see the full text article.

2012 Class. Quantum Grav. 29077001

(http://iopscience.iop.org/0264-9381/29/7/077001)

View the table of contents for this issue, or go to the journal homepage for more

Download details:

IP Address: 129.123.124.160

The article was downloaded on 19/03/2012 at 20:26

Please note that terms and conditions apply. 
NOTE

\title{
All homogeneous pure radiation spacetimes satisfy the Einstein-Maxwell equations
}

\author{
C G Torre \\ Department of Physics, Utah State University, Logan, UT 84322-4415, USA
}

Received 8 February 2012

Published 14 March 2012

Online at stacks.iop.org/CQG/29/077001

\begin{abstract}
It is shown that all homogeneous pure radiation solutions to the Einstein equations admit electromagnetic sources. This corrects an error in the literature.
\end{abstract}

PACS numbers: 04.20.-q, 04.20.Jb, 04.40.Nr

A spacetime $(M, g)$ is said to be a homogeneous pure radiation solution of the Einstein equations if it admits a transitive group of isometries and if there exists a function $\Phi$ and a 1 -form $k$ such that the Einstein tensor $G$ is of the form

$$
G_{a b}=\Phi^{2} k_{a} k_{b}, \quad \text { where } g^{a b} k_{a} k_{b}=0 .
$$

A classification of homogeneous pure radiation solutions is given in [1] based upon results of [2] and [3]. Such solutions are all pp waves, and they are either plane waves or are diffeomorphic to

$$
g=\mathrm{d} x \otimes \mathrm{d} x+\mathrm{d} y \otimes \mathrm{d} y+\mathrm{d} u \odot \mathrm{d} v-2 \mathrm{e}^{2 \rho x} \mathrm{~d} u \otimes \mathrm{d} u, \quad \rho=\text { const. }
$$

The plane waves are known to arise from electromagnetic sources. It is asserted in [1, 3], based upon results of [4], that the metric (2) does not arise from an electromagnetic source. However, the metric (2) does arise from an electromagnetic source. In fact, infinitely many electromagnetic fields can serve as the source for the spacetime defined by (2).

For any function $f(u)$ the null 2-form

$$
F=2 \rho \mathrm{e}^{\rho x}[\cos (\rho y+f(u)) \mathrm{d} u \wedge \mathrm{d} x-\sin (\rho y+f(u)) \mathrm{d} u \wedge \mathrm{d} y],
$$

its Hodge dual $\tilde{F}$ and the metric (2) satisfy the source-free Maxwell equations $d F=0=d \tilde{F}$ and the Einstein equations:

$$
G_{a b}=\frac{1}{2}\left(F_{a c} F_{b}^{c}+\tilde{F}_{a c} \tilde{F}_{b}^{c}\right)=4 \rho^{2} \mathrm{e}^{2 \rho x} \nabla_{a} u \nabla_{b} u .
$$

The electromagnetic field given in (3) is 'non-inheriting', that is, it is not invariant under the isometry group of $(M, g)$. For example, $F$ is not invariant under translations in $y$ for any choice of $f(u)$.

Thus, granted theorem 12.6 of [1], all homogeneous pure radiation solutions of the Einstein equations arise from electromagnetic sources. 


\section{Acknowledgments}

I thank Ian Anderson for discussions. These results were obtained using the DifferentialGeometry package in Maple.

\section{References}

[1] Stephani H, Kramer D, MacCallum M, Hoenselaers C and Herlt E 2003 Exact Solutions of Einstein's Field Equations 2nd edn (Cambridge: Cambridge University Press)

[2] Wils P 1989 Class. Quantum. Grav. 61243

[3] Steele J 1990 Class. Quantum. Grav. 7 L81

[4] Sippel R and Goenner H 1986 Gen. Rel. Grav. 181229 